\title{
Liver Cysts in a Kitten with Platynosomum sp.
}

\author{
Isabela das Neves Piana ${ }_{\oplus}$, Alexandre Coltro Gazzone ${ }_{\oplus \infty}$, Luciana Lopes Simplício \\ Mariana Isa Poci Palumbo \& Veronica Jorge Babo-Terra
}

\begin{abstract}
Background: Hepatic cysts are rarely described in association with infections by Platynosomum sp. Infected animals are most often asymptomatic, and the severity of symptoms is associated with the number of biliary tract parasites, which may lead to cholangitis and cholangiohepatitis. Although platinosomiasis is often associated with cholangitis and cholangiohepatitis, it rarely is with polycystic disease. For the parasite's life cycle to occur, the infected cat must eliminate the eggs in the feces and three intermediate hosts are needed: snails, terrestrial isopods and vertebrates like the frog or the gecko. The eggs are ingested by the snails, then the miracids are released and matured into the mother sporocyst form, which originates child sporocysts containing the cercariae, that leave the mollusks for the soil and are ingested by the terrestrial isopod in which the cercariae matures until metacercariae. Vertebrates ingest terrestrial isopods and are ingested by felines. The present study aimed to report an unusual case of platinosomiasis with the development of multiple hepatic cysts.

Case: A mixed breed male kitten was admitted with a history of apathy, hyporexia, increased abdominal volume and jaundice. In the ultrasound examination, we could see hepatomegaly and several hypoecogenic rounded structures, similar to cysts. There was an increase of serum concentration of the hepatic enzymes alanine transaminase and gamma glutamyl transferase enzyme. The parasite's eggs were investigated in the patient's feces using the simple sedimentation method, with a negative result. The animal was submitted to celiotomy and it was possible to observe several cystic structures in the liver. The cysts content was sent to cytology and culture. Cytology result was compatible with liver cyst and there was no bacterial growth in the culture. Bile fluid was collected and sent for Platynosomum sp. research using the centrifugal sedimentation test in formalin-ether solution, which allowed the parasite's eggs to be observed. The cat was treated with praziquantel, silymarin, S-Adenosyl methionine, and ursodeoxycholic acid. The patient gradually improved from jaundice and there was a reduction in abdominal volume.

Discussion: This report describes a case of platinossomiasis associated with polycystic liver disease in a domestic cat, which seems to be an uncommon presentation. Most infected cats are asymptomatic, but some animals may exhibit anorexia, apathy, increased abdominal volume due to hepatomegaly and/or ascitis and jaundice. Although infestation in domestic cats is relatively common, its association with liver cysts is rare or poorly reported in the literature, representing a diagnostic challenge, which makes mandatory the inclusion of this differential diagnosis in polycystic liver diseases in cats. The diagnosis of this parasitosis can be made based on the association among clinical signs, laboratory tests and ultrasound examination, but the definitive diagnosis is usually made by visualizing the parasite's eggs. In the case described, it was not possible to observe parasite's eggs in the patient's feces, but in the bile. Platynosomum sp. infection should always be considered as a differential diagnosis in cases of polycystic liver disease in cats, especially in countries with tropical or subtropical climate. Early diagnosis and appropriate treatment were fundamental for the improvement of the patient's clinical condition.
\end{abstract}

Keywords: feline, liver, platinosomiasis, polycystic disease, jaundice, cystic lesions. 


\section{INTRODUCTION}

Hepatic cysts are a rare condition in felines and their pathogenesis is still unclear. They can be congenital or acquired and, when congenital, most are from the bile duct and are present as part of a polycystic disease that affects several organs [3]. Congenital cysts are usually multiple and associated with feline polycystic disease, more frequent in Persian cats [2]. Acquired hepatic cysts can be single or multiple and usually occur secondary to trauma, inflammation, neoplasia and, in rare cases, caused by hepatic trematodes [3].

Platynosomum sp. is the most important liver parasite in domestic cats and it is commonly associated with cholangiohepatitis, one of the main conditions that affects cats [10]. Most animals do not have or show few clinical signs, but in the most severe infections, bile duct obstruction can occur, leading to cholangitis and possible liver cirrhosis [4]. Some clinical signs that can be observed are: emesis, anorexia, apathy, increased abdominal volume, diarrhea and jaundice [9].

Although platinosomiasis is often associated with cholangitis and cholangiohepatitis, it rarely is with polycystic disease [5]. In a case reported, the cat showed severe infestation by the trematode which led to acquired polycystic disease [11]. Ultrasonography can reveal tortuosity and dilation of the bile ducts, while biochemical examination often reveals an increase in liver enzymes [8]. The most used treatment for this disease is praziquantel in dosages of 20 to $30 \mathrm{mg} / \mathrm{kg}$ every $24 \mathrm{~h}$, for 5 days [7]. Thus, we describe here an unusual case of platinosomiasis attended at the Federal University of Mato Grosso do Sul (UFMS).

\section{CASE}

A mixed breed male kitten was admitted to the Veterinary Hospital of the Federal University of Mato Grosso do Sul with a history of apathy, hyporexia, increased abdominal volume and jaundice. The patient had been rescued a few days ago, so the guardian was not able to inform the exact beginning of the clinical signs. An informed consent has been obtained from the client to use the animal in this report.

On physical examination, temperature, heart rate, respiratory rate and pulse were within the range considered normal for the species. The patient was apathetic, jaundiced and it was possible to observe an increase in abdominal volume (Figure 1A). In the ultrasound examination, we could see hepatomegaly and several hypoecogenic rounded structures, similar to cysts, but as they elongated to the movement of the ultrasound cut, the image could not distinguish dilated bile ducts from hepatic cysts (Figure 1B).

The total blood count was within normal range. There was an increase of serum concentration of the hepatic enzymes alanine transaminase (ALT: 931.5 UI/L; reference range: 28 to $83 \mathrm{UI} / \mathrm{L}$ ) and gamma glutamyl transferase enzyme (GGT: $33.8 \mathrm{UI} / \mathrm{l}$; reference range: 1.3 to $5.1 \mathrm{UI} / \mathrm{L})$. Creatinine and albumin were within normal range. The parasite's eggs were investigated in the patient's feces using the simple sedimentation method, with a negative result.

The animal was submitted to celiotomy for diagnostic and therapeutic purposes. During the procedure, it was possible to observe several cystic structures in the liver, which were drained (Figure 1C). The cysts content was sent to cytology and culture. Cytology result was compatible with liver cyst and there was no bacterial growth in the culture. Bile fluid was collected and sent for Platynosomum sp. research using the centrifugal sedimentation test in formalin-ether solution, which allowed the parasite's eggs to be observed.

The cat was treated with praziquantel ${ }^{1}[20$ $\mathrm{mg} / \mathrm{kg}$ once a day, for 5 days]. Long treatment with silymarin $^{1}[50 \mathrm{mg} / \mathrm{kg}$ once a day], S-Adenosyl methionine $^{1}$ [90 $\mathrm{mg}$ once a day] and ursodeoxycholic $\operatorname{acid}^{2}$ [15 mg/kg once a day] was also recommended. The patient gradually improved from jaundice and there was a reduction in abdominal volume, so that 3 months later the cat completely recovered and is still under observation for recurrencies.

\section{DISCUSSION}

This report describes a case of platinossomiasis associated with polycystic liver disease in a domestic cat, which seems to be an uncommon presentation. Most infected cats are asymptomatic, but some animals may exhibit anorexia, apathy, increased abdominal volume due to hepatomegaly and/or ascitis and jaundice [9], which were quite the same signs this cat showed. Some authors have also cited weight loss, leading to anemia and even death [1]. An author reported a 10-year-old male mongrel feline with an obstruction of the biliary tract caused by Platynosomum sp. infection [6]. Our patient showed increased abdominal volume due to the numerous liver cysts and did not presente ascitis. 


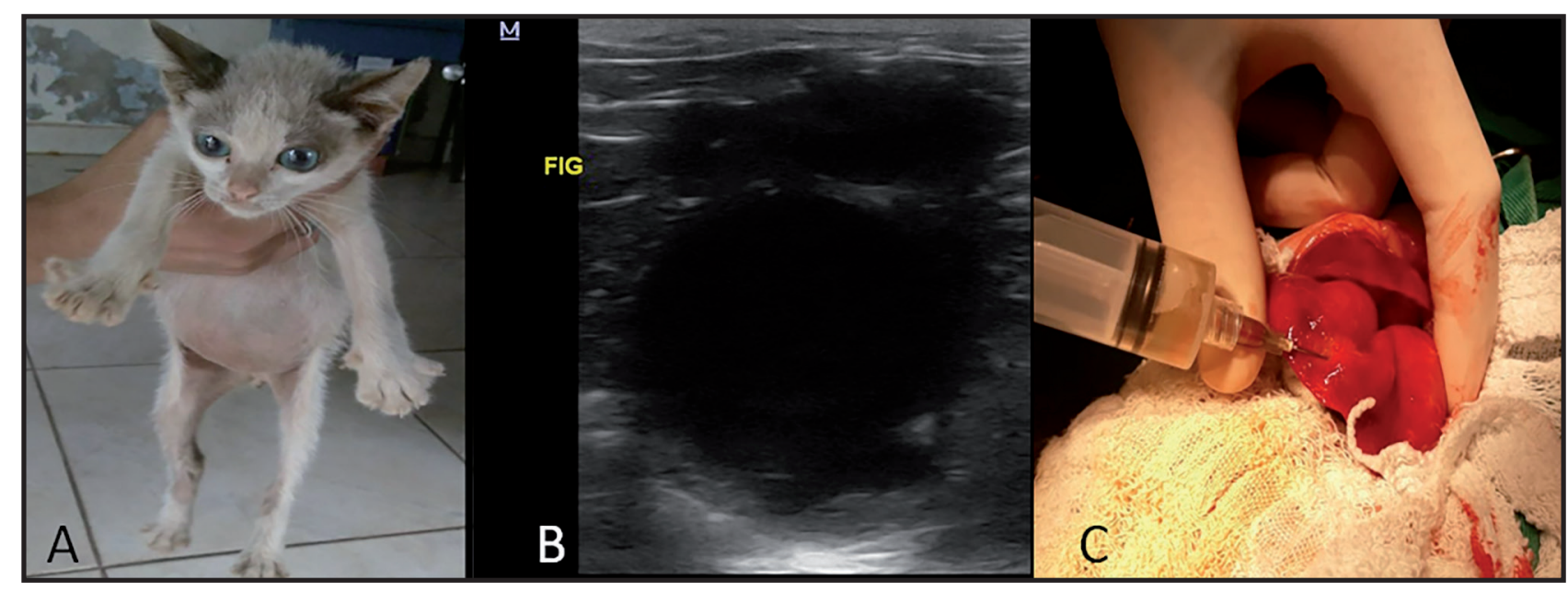

Figure 1. A- One mixed breed male kitten with increased abdominal volume. B- Rounded structures observed in ultrasound examination of the liver. C- Hepatic cysts being drained during the surgical procedure.

Hepatic cysts can be classified as congenital or acquired. Since the patient was young, we could initially assume it might be congenital, but once the history of the disease was uncertain it was necessary to include other causes of cystic liver disease in the differential diagnoses, such as trauma, parasitism or inflammation [3]. Due to the age and free living style of the kitten, its clinical signs were suggestive of Platynosomum sp. infection.

The ultrasound examination of patients suspected of platynosomiasis may reveal dilation and tortuosity of the bile ducts [8]. In our case, we initially suspected of dilated bile ducts, but round structures observed led us to think also in cysts. Ultrasound findings of infected cats are usually present in advanced stages and may be dependent on the parasite load. Alterations in the bile ducts were observed in $67 \%$ of the cases in a previous study [8], which was not in accordance with our findings. This alteration is possibly a consequence of the inflammatory process or to partial bile duct obstruction caused by the parasites, which was not present in our case.

Cystic lesions observed in feline polycystic disease are usually seen in several organs, mainly the kidneys [2]. In the case described, it was not possible to observe cysts in other organs. In addition, due to the country's climate and others reports registered in Brazil [5,11], the clinical suspicion of Platynosomum sp. infestation was considered.

The diagnosis of this parasitosis can be made based on the association among clinical signs, laboratory tests and ultrasound examination, but the definitive diagnosis is usually made by visualizing the parasite's eggs [3]. Despite the possibility of detecting eggs through a fecal examination, this test is not completely effective in confirming an infestation: only $25 \%$ of infested cats are identified using this method and serial repetitions are often necessary [1]. In the case described, it was not possible to observe parasite's eggs in the patient's feces, but in the bile.

The animal was treated with praziquantel [20 $\mathrm{mg} / \mathrm{kg}$ once a day for 5 days], as recommended by other authors [7] and required a subsequent treatment protocol 3 months after. The prognosis of this affection is considered unfavorable since chronic infection and intra and extra hepatic obstructions lead to further destruction of the parenchyma and possible future liver failure [5]. However, as far as we are aware, the cat is in good general clinical condition.

In conclusion, Platynosomum sp. infection should always be considered as a differential diagnosis in cases of polycystic liver disease in cats, especially in countries with tropical or subtropical climate. Early diagnosis and appropriate treatment were fundamental for the improvement of the patient's clinical condition. The cat continues to be monitored and, up to the time of this report, he is in good health.

\section{MANUFACTURERS}

${ }^{1}$ Drogavet Farmácia de manipulação. Campo Grande, MS, Brazil. ${ }^{2}$ Zambon Laboratórios Farmacêuticos Ltda. São Paulo, SP, Brazil.

Declaration of interest. The authors report no conflicts of interest. The authors alone are responsible for the content and writing of the paper. 


\section{REFERENCES}

1 Azevedo F.D., Veiga C.C.P., Scott F.B., Correia T.R., Fernandes J.I. \& Verocai G.G. 2013. Avaliação radiográfica e ultrassonográfica do fígado e da vesícula biliar em gatos domésticos (Felis catus domesticus) parasitados por Platynosomum illiciens (Braun, 1901) Kossak, 1910. Revista Brasileira de Medicina Veterinária. 35: 283-288.

2 Bosje J.T., van den Ingh T.S.G.A.M. \& van der Linde-Sipman J.S. 1998. Polycystic kidney and liver disease in cats. The Veterinary Quarterly. 20: 136-139.

3 Bunch S.E. 2009. Hepatobiliary diseases in the cat. In: Nelson R.W. \& Couto G.C. (Eds). Small Animal Internal Medicine. St. Louis: Elsevier, pp.520-540.

4 Carreira V.S., Vieira R.F.C., Machado G.F. \& Luvizotto M.C.R.2008. Feline cholangitis/cholangiohepatitis complex secondary to Platynosomum fastosum infection in a cat. Revista Brasileira de Parasitologia Veterinária. 17: 184-187.

5 Daniel A.G.T., Diaz R.F., Camignatto L.O., Kage N.K., Pellegrino A. \& Cogliati B. 2012. Polycystic liver disease associated with Platynosomum fastosum infection in a cat. Braz. Journal of Veterinary Pathology. 5: 137-141.

6 Facin C.A., Montanhim L.G., Sfrizo S.L., Camplesi C.A., Dias G.G.G.L. \& Moraes C.P. 2018. Resolution of a biliary obstruction caused by Platynosomum fastosum in a feline by a modified cholecystoduodenostomy approach case report. Acta Veterinaria-Beograd. 68(2): 224-231.

7 Norsworth G.D. 2006. Flukes: Liver, biliary and pancreatic. In: The Feline Patient. Ames: Blackwell, pp.108-109.

8 Salomão M., Souza-Dantas L.M., Mendes-de-Almeida F., Branco A.S., Bastos O.P.M., Sterman F. \& Labarthe N. 2005. Ultrasonography in hepatobiliary evaluation of domestic cats infected by Platynosomum looss. International Journal of Applied Research and Veterinary Medicine. 3: 271-279.

9 Sampaio M.A.S., Berlim C.M., Angelim A.J.G.L., Gondim L.F.P. \& Almeida M.A.O. 2006. Infecção natural pelo Platynosomum looss 1907, em gato no município de Salvador, Bahia. Revista Brasileira de Saúde e Produção Animal. 7 (1):1-6.

10 Vieira A.L.S., Ecco R., Lima W.S. \& Guedes R.M.C. 2009. Platynosomum fastosum infection in two cats in Belo Horizonte, Minas Gerais State. Brazilian Journal of Veterinary Pathology. 2: 45-58.

11 Xavier F.G., Morato G.S., Righi D.A., Maiorka P.C. \& Spinosa H.S. 2007. Cystic liver disease related to high Platynosomum fastosum infection in a domestic cat. Journal of Feline Medicine and Surgery. 9: 51-55. 